\title{
Glossar zu methodologisch relevanten Begriffen
}

Das Glossar erläutert in Auswahl und Kurzform zentrale Begriffe der Studie, wie sie im Kontext der Argumentation verstanden und gegebenenfalls modifiziert werden.

Aisthetische Historiografie: Begriffssetzung, um die Form der untersuchten $\rightarrow$ medialen Geschichtsbildmodellierung zu fassen. Aisthetik wird von dem Technikphilosophen Gernot Böhme (2001) aufgegriffen, welcher Konzepte ästhetischer Wahrnehmung auf lebensweltliche Zusammenhänge überträgt. Die vorliegende Studie untersucht insofern medienästhetische Konfigurationen, die zeitliche Differenzverhältnisse (eines Vorher/Nachher) räumlichpräsentisch vermitteln. $\rightarrow$ Kap. $5.8 ; \rightarrow$ Performance; $\rightarrow$ Erfahrung

Archäologie/Medienarchäologie: Begrifflichkeit von Michel Foucault zum Verhältnis von Geschichte und ihrer Repräsentation. Unter dem Eindruck der digitalen Domäne skizziert Thomas Elsaesser (2004) im Anschluss an Foucault eine Medienarchäologie als historiografisches Modell, das sich von einem strikt konsekutiv-chronologischen Ordnungsprinzip abwendet zugunsten von sich verzweigenden Netzwerken und achronologischen Zusammenhängen. $\rightarrow$ Kap. 3.2; $\rightarrow$ aisthetische Historiografie

Bridging the gap, marking the difference: Ein von dem Filmwissenschaftler Frank Kessler (2011) zur Beschreibung von zeitlichen Relationen profiliertes Konzept, das bei der Kuratierung und Aufführung von frühem Film heute zum Tragen kommt. Das Konzept wird nun wahrnehmungstheoretisch gewendet, es schließt an das Prinzip koexistierender Schichtungen an. $\rightarrow$ Kap. 5.4; $\rightarrow$ Cluster; $\rightarrow$ aisthetische Historiografie; $\rightarrow$ Performance

Cluster: Die Fallstudien zu $\rightarrow$ Reprisen von historischen Filmen werden als clusterförmig strukturierte Komplexe angegangen; diese begriffen als beziehungssetzende Wahrnehmungskonfigurationen, die über kopräsente Schichtungen unterschiedlicher Zeitebenen funktionieren. $\rightarrow$ Kap 3.9.2; $\rightarrow$ Erfahrung; $\rightarrow$ Dauer

Dauer (la durée): Philosophisches Konzept von Henri Bergson (1991/1896) zur subjektiv erlebten Zeit als einem kontinuierlichen Fluss. Die Dauer 
unterscheidet sich von der temps, die physikalisch messbar ist. Dauer bedeutet ständige Veränderung (changement) und ist gedächtnishaft. $\rightarrow$ Kap. 4.1; $\rightarrow$ Erfahrung

Digitale Dispositive: Der Medienwissenschaftler Jan Distelmeyer (2012) hat ausgehend von Jean-Louis Baudry DVDs und Blu-Ray-Discs als digitale Dispositive theoretisiert. Der analytische Fokus richtet sich auf die räumlichen wie technischen Voraussetzungen des Erscheinens von Filmen. Übertragung der Ansätze auf jüngere Anordnungen, in denen Filme zugänglich werden (unter anderem VoD, Internet): Dies bedingt die Problematisierung fester $\mathrm{Zu}$ schreibungen von Rezeptionsbedingungen und ihren Effekten auf Publikumssubjekte. $\rightarrow$ Kap. 5.6; $\rightarrow$ Kap. 7.3; $\rightarrow$ Performance; $\rightarrow$ Mise en Relation

Digitalisierung: Hat die Filmproduktion, -rezeption und -distribution auf allen Ebenen verändert. Schon Lev Manovich (2001) weist auf die methodologisch notwendige Differenzierung der Ebenen hin: Ursprünglich bezeichnet Digitalisierung den Prozess der Konversion von analog zu digital, die Umwandlung in binäre Codes. Die vorliegende Studie untersucht filmästhetische Phänomene und Dispositive als Ergebnisse von interessegeleiteten Interpretationen der numerischen Repräsentationen, in denen soziokulturelle Praktiken, (dominante) Denkmuster und (Zukunfts-)Phantasien kontextgebunden historiografisch wirksam werden. $\rightarrow$ Kap. $1 ; \rightarrow$ Kap. 2

\section{Enunziation (bei Bonusmaterialien/filmhistorischen Dokumentationen):}

Bezeichnet in Anlehnung an die struktural-linguistische Begrifflichkeit (Émile Benveniste) die Ebene des konkreten kommunikativen Äußerungsaktes, auf der sich Modi der Adressierung des Rezipienten textuell einschreiben. In filmhistorischen Dokumentationen tritt - in meiner Adaption des semio-pragmatischen Archivbilddiskurses ( $\rightarrow$ Lektüremodi) - Geschichte ästhetisch und dispositivisch als unpersönliche, abstrakte Äußerungsinstanz in Erscheinung. Filmhistorische Dokumentationen in digitalen Dispositiven werden zudem als Bonus vermarktet. Dieses Mehrwertversprechen beeinflusst die historiografisch wirksame Rezeptionserwartung des Zuschauers. $\rightarrow$ Kap. 6

Erfahrung: Ein für phänomenologisch ausgerichtete Wahrnehmungstheorie in Anschlag gebrachter Begriff (Morsch 2010), der mit Rekurs auf Gilles Deleuze Repräsentation sowie Subjektivität jenseits von psychoanalytischen und kognitiven Modellen verortet. Verzeitlichte Vollzugsprozesse werden 
zentral für filmästhetische Wahrnehmung, was mit Fragen des Körpers verknüpft wird: Erfahrung wird mit einer vom Film performativ hergestellten wie gleichzeitig vom Zuschauer mitkonstituierten Wahrnehmung identifiziert. Insofern wird audiovisuelle Historiografie als körperlicher (Nach-) Vollzug performativ konzipiert. $\rightarrow$ Kap. 5.2; $\rightarrow$ aisthetische Historiografie; $\rightarrow$ Performance; $\rightarrow$ Mise en Relation

Erfahrungskonsum: Begriffliche Anlehnung an die Soziologin Eva Illouz (2011/2009), die das Konzept der $\rightarrow$ Warenästhetik (Haug 2009/1971) aktualisiert. Sie untersucht die Funktion von Emotionen in der Konsumsoziologie. Übertragung auf das kulturindustrielle Produkt Film: Erfahrungskonsum wird als eine sinnlich-emotionale An- und Einbindung verstanden, die die Filmerfahrung in der digitalen Reprise zusätzlich prägen kann. $\rightarrow$ Kap. 6.6; $\rightarrow$ Imaginäres; $\rightarrow$ Warenästhetik; $\rightarrow$ aisthetische Historiografie

Fetischisierung: Anschluss an Hartmut Böhmes Kulturanalyse: Spezifische mediale Praktiken, die eine fetischisierende Wahrnehmungshaltung (Empfindung von „erregendem Zauber“) von digitalisierten Filmen aus dem Archiv und digitalen Dispositiven zur Folge haben. Fetischisierte Objekte müssen einerseits performativ-rituell dem Betrachter präsent gehalten werden, andererseits müssen sie gleichzeitig in einer Distanz verbleiben, sich dem Zugriff entziehen. Diese zweisträngigen Rezeptionsbedingungen sind zentral für mediale Formen, die Filmgeschichte fetischisieren. $\rightarrow$ Kap. 4.3; $\rightarrow$ Warenästhetik; $\rightarrow$ wechselseitige Bilder

Filmedition (DVD, Blu-Ray-Disc, Internet): Der Gebrauch der Begriffe Edition/Re-Edition ist den Marktpraktiken der Filmdistribution auf digitalen Trägern entlehnt, bei denen bisher keine verbindlichen Systematiken vorliegen, wie sie schon lange in der Literaturwissenschaft existieren (Ausnahmen: Drubek-Meyer/Izvolov 2007; Keitz 2013; Bohn 2013b; sie haben verschiedene philologische Ansätze zur Übertragung auf filmische Medien aufgezeigt). Um die Verwendung von Re-Edition jenseits editionswissenschaftlicher Normen zu markieren, ist die kompositorische Schreibweise mit Bindestrich gewählt; dies ist zugleich Hinweis auf die zeitliche Schichtung aus Wiederaufnahme und Neuschöpfung. Diese von amtlichen Regeln bewusst abweichende Schreibweise wird auch bei ähnlichen Kompositbegriffen wie etwa Re-Montage oder Re-Kontextualisierung verwendet. $\rightarrow$ Kap. 3.9.2 
Imaginäres/Imagination: Mehrschichtiger Begriffskomplex in der Studie; allgemein verstanden als Synonym des Fiktiven; die filmische Erzählung als Fiktion, die eine imaginäre Welt zur Anschauung bringt. Filmtheoretisch: Nach Christian Metz ist der Film für den Zuschauer ein imaginärer Signifikant: Bilder und Töne, die konkret wahrnehmbar, aber zugleich nur mimetische Substitute real abwesender Personen/Objekte sind. Filmanalytisch/Bild-(Inhalts-)Ebene: Ästhetische Strategien, die in Restaurierungsdokumentationen das Phantastische ausstellen und damit das Technoimaginäre des Digitalen speisen ( $\rightarrow$ Imagologie der Technik). Metatheoretische Dimension: Bei Hayden White (2008/1973) kommt der Vorstellungskraft eine wichtige Funktion für die Organisation von geschichtsbildenden Weltentwürfen zu. Warenästhetik und das Imaginäre: $\rightarrow$ Erfahrungskonsum; $\rightarrow$ Fetischisierung. Grundsätzlich: $\rightarrow$ Kap. 6

Imagologie der Technik (auch Technoimagination): Vor allem von dem Kulturwissenschaftler Hartmut Böhme skizziertes Forschungsfeld, das mythisierende Leitbilder der Technik, technische Phantasien untersucht, die heute in Massenmedien Ausdruck finden. Beispiele im Kontext von digitalen Technologien sind häufige Referenzen auf Magie und Zauberei. $\rightarrow$ Kap. 2.9; $\rightarrow$ Imaginäres; $\rightarrow$ Fetischisierung

Konsekration/(Kunst-)Wert: Nach dem Kultursoziologen Pierre Bourdieu (2011c/1977) geht es in der kulturellen Produktion um die Akkumulation von symbolischem Kapital; Prestige und Autorität fungieren als Währung. Marken-, Signatureffekte, Personen können Dingen ,Wert' verleihen, was Konsekrationskapital bedeutet (Konsekration $=$ Weihung). In wahrnehmungstheoretischer Perspektive wird dies als erfahrene Wertzuschreibung verstanden. $\rightarrow$ Kap. 7.2; $\rightarrow$ aisthetische Historiografie; $\rightarrow$ Warenästhetik; $\rightarrow$ Fetischisierung;

$\rightarrow$ Erfahrungskonsum; $\rightarrow$ Erfahrung

\section{Lektüremodi (hier auch historisierende Lektüremodi): Bestimmung} des Verhältnisses zwischen Bewegtbildern, ihren Referenzdimensionen und Rezeptionsmodi; dies angelehnt an semio-pragmatische Ansätze von Roger Odin. Mit dem Konzept ist kein prinzipieller Gegensatz zwischen Dokumentar-, Fiktions- oder Archivbildern anzusetzen: Die ontologische Frage, ob Filmbilder tatsächlich eine (vergangene) Wirklichkeit abbilden, verlagert sich auf die Frage nach dem Lektüremodus. Dieser fußt auf der Zuschreibung, welche Äußerungsinstanz den Bildern attribuiert wird. $\rightarrow$ Kap. 3.6; $\rightarrow$ Enunziation 
Mediale Geschichtsbildmodellierung: Formel, die den AV-medienanalytischen und wahrnehmungstheoretischen Fokus der Studie betont, dass nicht von Geschichtsschreibung im Horizont einer disziplinären Geschichtswissenschaft gesprochen wird. $\rightarrow$ Kap. 3 ; $\rightarrow$ aisthetische Historiografie; medialen Geschichtsbildmodellierungen wird im Kontext der digitalen Medientransition im Sinne einer Public History (Haber 2011) historiografische und erinnerungskulturelle Wirksamkeit zugeschrieben. $\rightarrow$ Kap. 6.1

Mediatisierte Erinnerung: Kulturwissenschaftlicher Problemkomplex zum Verhältnis von (Massen-)Medien und Erinnerungen. Nach Dijk (2007) findet eine wechselseitige Konstruktion statt - zwischen der autobiografischen Identitätsbildung des Subjekts und der Dynamisierung der Kategorien von privat/persönlich und öffentlich/kollektiv. Dijks Überlegungen werden wahrnehmungstheoretisch gewendet. $\rightarrow$ Kap. $3.8 ; \rightarrow$ prothetische Erinnerung; $\rightarrow$ aisthetische Historiografie

Memophänomenal: Beschreibung der wirkungsästhetischen Dimension von Affekt- und Erinnerungsbildern (wechselseitige Bilder), die bei $\rightarrow$ Reprisen historischer Filme in der digitalen Domäne zur Vermittlung der $\rightarrow$ Warenästhetik eingesetzt werden. Die zeitlich heterogene Wirkungsdimension zwischen Präsenz/Unmittelbarkeit und Vergangenheitseindrücken spielt mit individuellen wie kollektiven Erinnerungsprozessen. $\rightarrow$ Kap. 4.8

Memopolitik: Helvetischer Begriff für Gedächtnispolitik, der im Rahmen einer Initiative zur Pflege des nationalen Erbes mit Blick auf die fortschreitende Digitalisierung geprägt wurde. Er wird in dieser Studie gegenüber äquivalenten Komposita bevorzugt. $\rightarrow$ Kap. 2.8 und die nachfolgenden Unterkapitel

Mise en Relation: Begriffssetzung zu den Implikationen der Versionenhaftigkeit von Film. Mise en Relation wird verstanden als Gestaltungsform sowie als Erwartung, Wahrnehmungsakt und -erfahrung; Formen, die sowohl innerhalb von Filmen (z. B. in filmhistorischen Dokumentationen) als auch auf der Ebene des Dispositivs (z. B. Ästhetik der Menüstruktur) die Relativität mediengeschichtlicher Zusammenhänge ausstellen. $\rightarrow$ Kap. 7; $\rightarrow$ vergleichendes Sehen; $\rightarrow$ Konsekration/(Kunst-)Wert; $\rightarrow$ digitale Dispositive; $\rightarrow$ Erfahrung 
Passagen: Begrifflicher Rekurs auf Christina Scherers filmwissenschaftliche Ausführungen (2001) zu Walter Benjamin: Erinnerung und Geschichtsschreibung werden als archäologische Tätigkeiten beschrieben, die dem Modell einer zeitlichen Schichtung und dem Prinzip von Erkennbarkeit im Fluss (der Zeit) verpflichtet sind. Scherer sieht hier filmische Strukturen des Fragmentarischen und Heterogenen im Zusammenspiel mit der wirkungsästhetischen Dimension - etwa mit dem Erinnerungsvermögen des $\mathrm{Zu}$ schauers. $\rightarrow$ Kap. 3.9

Performance (auch digitale Performance): Vielschichtig gebrauchter Begriff: Methodologisch schließt performative Sinnproduktion an pragmatische $\mathrm{Zu}-$ gänge an. Der Anglizismus assoziiert technische Prozessleistungen von Tools und Programmen. $\rightarrow$ Kap. 5

Prothetische Erinnerung: Die Formulierung (Landsberg 2004/1996, Elsaesser 1996b, Burgoyne 1999) beschreibt Erinnerungen in massenmediatisierter Form, die sich nicht auf selbst er-/gelebte Momente gründen, aber trotzdem biografisch-identitätsbildend auf das Subjekt wirken. $\rightarrow$ Kap. 6.7; $\rightarrow$ mediatisierte Erinnerungen; $\rightarrow$ memophänomenal; $\rightarrow$ aisthetische Historiografie

Remastered: Vermarktungslabel für die digitale $\rightarrow$ Re-Edition filmischer Werke. Trotz einiger Definitionsversuche wurde der Begriff in den ersten Dekaden der 2oooer Jahre diffus eingesetzt: Er bezeichnet keinen technisch standardisierten Vorgang; zugleich ist er aber umstandslos wertsteigernd konnotiert. Implizit wird ein teleologischer Fortschrittsgedanke von Mediengeschichte transportiert. $\rightarrow$ Kap. 3.1; $\rightarrow$ Warenästhetik; $\rightarrow$ Fetischisierung

Reprise (dt. hier Wiederaufnahme): Begriffskomplex des Filmwissenschaftlers François Niney (2012/2009), der pointiert zur Analyse der soziokulturellen Folgen der Filmdigitalisierung herangezogen wird; bezeichnet sowohl Prozesse der Wiederaufnahme eines präexistenten Phänomens als auch eine Erprobung des Materials hin auf Bedeutungspotenziale in neuen Kontexten. $\rightarrow$ Kap. 3.9

Update: Titelgebendes Prinzip, das die technologischen, ökonomisch orientierten Politiken der Transformation heutiger digitaler Medienumgebungen zugespitzt fasst; ideologisch-diskursiv geprägt von der Vorstellung einer ständigen Verbesserung. $\rightarrow$ Kap. $1 ; \rightarrow$ Remastered 
Vergleichendes Sehen: Von dem Kunsthistoriker Heinrich Dilly (unter Rekurs auf Heinrich Wölfflin) reflektiertes Verfahren: die methodischen Konsequenzen der vergleichenden (Dia-)Projektion zweier Bilder für die Kunstwissenschaft; Übertragung dieses Ansatzes auf Bildkorrelationen zur Veranschaulichung der Qualitäten von Digitalisaten: z. B. medial inszenierte Split-Screen-Konfigurationen, die Vorher/Nachher-Bilder in eine historiografisch und erinnerungspolitisch wirksame Beziehung zueinander setzen. $\rightarrow$ Kap. $7.11 ; \rightarrow$ Mise en Relation; $\rightarrow$ aisthetische Historiografie

Warenästhetik: Aktualisierte Begrifflichkeit nach Wolfgang Fritz Haugs Kritik der Warenästhetik (1971 und 2009) zur spezifizierten Analyse des ästhetischen Warencharakters von Filmen in der digitalen Domäne. $\rightarrow$ Kap. 4.2; $\rightarrow$ Fetischisierung; $\rightarrow$ wechselseitige Bilder; $\rightarrow$ memophänomenal; $\rightarrow$ Erfahrungskonsum

Wechselseitige Bilder: Wahrnehmungstheoretische Adaption von Gilles Deleuzes Überlegungen zu Affekt- und Erinnerungsbildern als temporal heterogene Phänomene (koaleszierend zwischen Aktuellem und Virtuellem). $\rightarrow$ Kap. $4.1 ; \rightarrow$ Fetischisierung; $\rightarrow$ memophänomenal; $\rightarrow$ mediale Geschichtsbildmodellierung 